Running Title: Family identification and loneliness

\title{
Family identification is a protective resource for people with eating disorders because it ameliorates feelings of loneliness
}

Niamh McNamara*1 ${ }^{1}$, Juliet R. H. Wakefield ${ }^{1}$, Tegan Cruwys ${ }^{2}$, Adam Potter ${ }^{1}$, Beth Jones ${ }^{1}$ \& Sara McDevitt ${ }^{3,4}$

${ }^{1}$ Department of Psychology, Nottingham Trent University, UK

${ }^{2}$ Research School of Psychology, The Australian National University

${ }^{3}$ Department of Psychiatry, University College Cork, Ireland

${ }^{4}$ Consultant Child \& Adolescent Psychiatrist, Health Services Executive, Ireland

* Corresponding author: Dr Niamh McNamara, Department of Psychology, Nottingham Trent University, 50 Shakespeare Street, Nottingham NG1 4FQ

\section{Acknowledgements}

The authors wish to thank the following organisations for their support of this research: Bodywhys; Health Service Executive National Clinical Programme for Eating Disorders, Ireland; SpunOut.ie; Eating Disorders Association Northern Ireland; The Laurence Trust; MQ Mental Health UK; Anorexia \& Bulimia Care, UK; First Steps ED, UK; MaleVoicED; South Yorkshire Eating Disorders Association, UK; National Eating Disorders Association USA; F.E.A.S.T.; Eating Disorders Research Center, USA.

The authors declare that they do not have any potential conflicts of interest to report.

This work was partly funded by a grant from the Irish National Office for Suicide Prevention and Bodywhys - The Eating Disorders Association of Ireland. 


\title{
Family identification is a protective resource for people with eating disorders because it ameliorates feelings of loneliness
}

\begin{abstract}
Families play an important role in eating disorder recovery. Recently, it has been suggested that they can ameliorate the loneliness and social isolation associated with an eating disorder. However, the psychological mechanisms through which this occurs have yet to be systematically explored. Utilising the Social Identity Approach to Health, we explore whether identification with one's family group positively predicts health and wellbeing in people with eating disorders due to its capacity to reduce feelings of loneliness. We investigate this in two studies ( $N=82 ; N=234)$, one of which was conducted in the early stages of the COVID-19 pandemic. In both studies, we demonstrated that family identification protects health. This was apparent in participants reporting fewer and less severe ED symptoms, and in the context of the COVID-19 pandemic, reduced eating disorder-related impact and reduced anxiety. In both studies, these benefits were attributable to the protective role of family identification against loneliness. Our findings provide a framework for understanding in general why family is so important in treatment, including in the case of adults. It also supports the focus in treatment on the family as a group rather than as a collection of interpersonal relationships.
\end{abstract}

Keywords: eating disorders, family functioning, social identity, COVID-19, loneliness 
Although loneliness is a significant issue for people with eating disorders (PWED), the complex relationship between social connections, loneliness, and ED symptoms remains relatively under-explored in the literature. This is problematic, especially given the increased urgency for such investigations in light of the COVID-19 pandemic. Virus-related social distancing has the capacity to exacerbate ED symptoms given it greatly increases the likelihood of loneliness (Rodgers et al., 2020; Fernández-Aranda et al., 2020; Cooper et al., 2020). In this paper we use the Social Identity Approach to Health (Haslam et al., 2018) to explore whether identification with one's family - an important social group for ED recovery, and one that was most likely to be accessible during social distancing restrictions - positively predicts PWED's health and wellbeing due to its capacity to reduce feelings of loneliness.

\section{Social isolation, loneliness, and eating disorders}

While social isolation typically refers to a scarcity of (objective) social ties, loneliness is a stressful emotional state whereby an individual subjectively perceives that their social network is deficient (Perlman \& Peplau, 1981). Social isolation and loneliness are robust predictors of poor health. Indeed, their effect on mortality risk has been found to be of comparable size to well-established lifestyle and environmental factors (Holt-Lunstad, et al., 2015). For PWED, poor social functioning, interpersonal difficulties, and a tendency to appraise the social environment as threatening contribute to distressing social experiences and social withdrawal (Cardi et al., 2018; Levine, 2012). Loneliness is especially problematic during the acute phase of EDs (Westwood et al., 2016). During recovery, loneliness can precede a return to disordered behaviours (Cardi et al., 2018; Cockell et al., 2004).

COVID-19 restrictions have reportedly intensified ED symptom severity (FernándezAranda et al., 2020; Phillipou et al., 2020). The loneliness associated with the shrinking of already-impoverished social networks might underlie symptom exacerbation (Rodgers et al., 
2020). This has been a difficult time for families living with an ED. PWED and their carers identified isolation as a key concern in the early stages of the pandemic; in particular, carers saw the potential for restrictions to increase carer burden and negatively impact family dynamics (Fernández-Aranda et al., 2020). However, other research suggested that some carers felt that increased time at home allowed them to provide more support to their loved one and spend more time together as a family (Clark Bryan et al., 2020). Similarly, some PWED reported that a sense of connection with family and friends was a positive change that occurred in the pandemic's early stages (Termorshuizen et al., 2020).

Of course, these issues of connection and loneliness are not only relevant during the COVID-19 pandemic: they are also core elements of PWED's social recovery (Patel et al., 2016). Understanding how to support social recovery requires a theoretical framework which can specify the mechanisms through which social relationships protect health and wellbeing. The Social Identity Approach to Health (Haslam et al., 2018) can offer such a framework.

\section{Group Memberships, Social Identities, and Eating Disorder Recovery}

Mirroring the distinction between social isolation and loneliness, the Social Identity Approach to Health argues that it is not our objective group memberships or social contact that account for the robust positive relationship between social connectedness and health (Haslam et al., 2018). Instead, it is the psychological experience of group membership, or social identification (Tajfel \& Turner, 1979), that is crucial for health (Haslam et al., 2018). When we identify as a member of a group, it informs our self-definition - and this is critical to a group's health-enhancing potential. Social identification allows one to benefit from the psychological resources provided by the group, such as a feeling of belonging, collective efficacy, personal control, and social support (Greenaway et al., 2015). More recently, it has been found that identification with one's local community and with friendship groups at 
university predicted better personal wellbeing (McNamara et al., under review) and lower levels of depression, anxiety, and paranoia (McIntyre et al., 2018) by decreasing feelings of loneliness.

The notion of the 'psychosomatic family' has been replaced with the view that the family is a vital recovery resource (Le Grange et al., 2009). Family involvement is a central component of most treatments for eating disorders that have a strong evidence base (such as FBT, FTAN, CBRE, MANTRA, etc.), as well as being a powerful general source of support and connectedness (McMaster et al., 2004). It has been suggested that families can ameliorate ED-related loneliness (Treasure \& Palazzo Nazar, 2016), however, the psychological mechanisms through which this occurs have yet to be systematically explored. We suggest that identification with family is crucial to experiencing these health benefits. Outside the ED context, family identification has been associated with positive mental health outcomes for adolescents (Miller et al., 2017), those affected by financial distress (Stevenson et al., 2020), and those affected by intimate partner violence (Naughton et al., 2015). However, the degree to which family identification can reduce loneliness (and whether this is one of the mechanisms for its health benefits) has yet to be explored for PWED.

\section{The Current Research}

In this paper, we report two cross-sectional studies designed to investigate the social psychological mechanisms through which family identification predicts health in PWED. We predicted that family identification would be associated with reduced ED symptom severity. We also predicted that this relationship would be mediated via reduced feelings of loneliness.

Study 1 examined these phenomena in a pre-COVID context among PWED who were in (or had recently received) treatment. Study 2 was conducted with PWED in the community during the early stages of the COVID-19 pandemic, following the introduction of social 
distancing measures. We predicted that these restrictions would lead to an exacerbation of ED symptoms and increased anxiety. Central to this is the loneliness associated with social distancing measures.

In Study 2, we proposed a serial mediation model, such that family identification predicted reduced feelings of loneliness, which in turn predicted reduced ED-related impact of COVID-19, which in turn predicted better health. We hypothesised that reduced feelings of loneliness will predict reduced ED-related impact of COVID-19 because it has been wellestablished within the Social Cure perspective (e.g., Haslam et al., 2018) that our social group memberships provide us with the support and resources we need to manage life's challenges (such as coping with COVID-19 in the context of an ED). Thus, experiencing low levels of loneliness (which itself is predicted by feeling connected to one's family) means that one is likely to have the social support needed in order to cope with the negative impacts of COVID restrictions. Finally, we hypothesise that reduced ED-related impact of COVID-19 will predict reduced anxiety and ED symptom severity because participants who experience fewer negative impacts during COVID-19 are likely to feel they can cope better with the situation, and are thus also less likely to need to engage in disordered eating as a way to cope with challenges. Ethical approval for both studies was granted by the first author's institutional Research Ethics Committee.

\section{Study 1}

Study 1 took place during July to October 2019 as part of an investigation into ED treatment experiences in Ireland. Participants were included if they were over 18 years, were living with or had recovered from an ED, and had received any form of ED-related service within the previous three years. 


\section{Participants and Procedure}

82 participants were recruited through online advertisements on the social media account and website of Bodywhys (The Eating Disorders Association of Ireland). This included 70 females, 1 male, 1 transgender (people who experience incongruence between gender assigned at birth and gender identity), 1 non-binary, 9 not reported. Participants ranged in age from 18 to 62 years $\left(M_{\text {age }}=28.95\right.$ years, $\left.S D=8.33\right)$. Of those who reported their living arrangements, $38 \%$ lived with their parents, $19 \%$ lived with a partner, $18 \%$ lived alone, $16 \%$ lived with other family members, and $8 \%$ lived with friends. $79 \%$ of participants reported receiving a formal diagnosis. The most common self-reported diagnosis was Anorexia Nervosa (AN; 50\%) followed by Bulimia Nervosa (BN; 19\%), Combination Anorexia Nervosa/Bulimia Nervosa (AN/BN; 9\%), Other Specified Feeding or Eating Disorder (OSFED; 9\%), Multiple diagnoses (6\%), Binge Eating Disorder (3\%), Avoidant/Restrictive Food Intake Disorder (ARFID; 2\%), and Pica (2\%). Of those who indicated their treatment status $(n=78), 62 \%$ were in treatment at the time of data collection, $24 \%$ had chosen to leave treatment, and $14 \%$ considered themselves fully recovered and were no longer in treatment.

\section{Measures}

Family identification was measured with Postmes et al.'s (2013) single-item group identification measure, which has been found to have comparable reliability and validity to longer scales (e.g., Reysen et al., 2013). Participants rated their agreement with the item ("I identify with members of my family") on a scale ranging from 1 ("Strongly disagree") to 7 ("Strongly agree").

Loneliness was measured with the Three-Item Loneliness Scale (Hughes et al., 2004). Participants rated their agreement with each statement (e.g., "How often do you feel that you lack companionship?") on a scale ranging from 1 (“Hardly ever") to 3 (“Often"). The sum of 
the items was found, with higher values indicating higher loneliness. In the general population, this measure has been found to be reliable $(\alpha=.72)$ and possesses both convergent and discriminant validity (Hughes et al., 2004).

ED symptom severity was measured with the sixteen-item shortened version of the Eating Attitudes Scale (EAT-16; McLaughlin, 2014). Participants were asked to think about the last month, and to rate their agreement with each item (e.g., "I am terrified about being overweight") on a scale ranging from 1 ("Never") to 6 ("Always"). The mean of the items was found, with higher values indicating more negative eating-related attitudes.

Finally, demographic information was recorded, including age, gender, whether the participant had received a formal eating disorder diagnosis, and the specific diagnosis received.

\section{Results}

\section{Descriptives and Correlations}

Descriptives and correlations for the variables can be found in Table 1. PWED who identified strongly with their family tended to feel less lonely $(p=.007)$ and have fewer negative eating-related thoughts and behaviours $(p=.03)$. These patterns remained when age, gender, and diagnosis (yes/no) were controlled for.

Table 1. Study 1: Means, standard deviations, alphas (where applicable), and correlations for the key variables

\begin{tabular}{lccccccc}
\hline Variable & Range & M & SD & $\alpha$ & 1 & 2 & 3 \\
\hline 1.Family Identification & $1-7$ & 4.27 & 1.86 & - & - & & \\
2.Loneliness & $3-9$ & 6.86 & 1.89 & .80 & $-.31^{* *}$ & - & \\
3.ED Symptom Severity & $1-6$ & 4.40 & 1.11 & .94 & $-.26^{*}$ & $.48^{* * *}$ & - \\
\hline Note: $* p<.05, * * p<.01, * * * p \leq .001$. & & & & &
\end{tabular}




\section{Mediation Analysis}

Model 4 in version 3.4 of Hayes' (2017) PROCESS macro was used to test the mediation model (see Figure 1). Analysis involved 5,000 bootstrapping samples with 95\% confidence intervals (LLCI/ULCI), using the percentile method, and controlled for gender, age, and diagnosis (yes/no). All reported coefficients are unstandardized.

Supporting predictions, there was a significant indirect effect of family identification on symptom severity though loneliness, Effect $=-.07$, Boot $S E=.03$, Boot LLCI $=-.15$, Boot $U L C I=-.01$. Family identification was a negative predictor of loneliness, Coeff $=-.30, S E=$ $.12, t=-2.54, p=.01, L L C I=-.53, U L C I=-.06$, while loneliness was a positive predictor of symptom severity, Coeff $=.25, S E=.07, t=3.82, p=.0003, L L C I=.12, U L C I=.38$. The total effect of family identification on symptom severity was negative and significant, Effect $=-.15, S E=.07, t=-2.19, p=.03, L L C I=-.29, U L C I=-.01$, and this became non-significant when loneliness was accounted for (direct effect), Effect $=-.08, S E=.07, t=-1.19, p=.24$, $L L C I=-.21, U L C I=.05$, indicating full mediation.

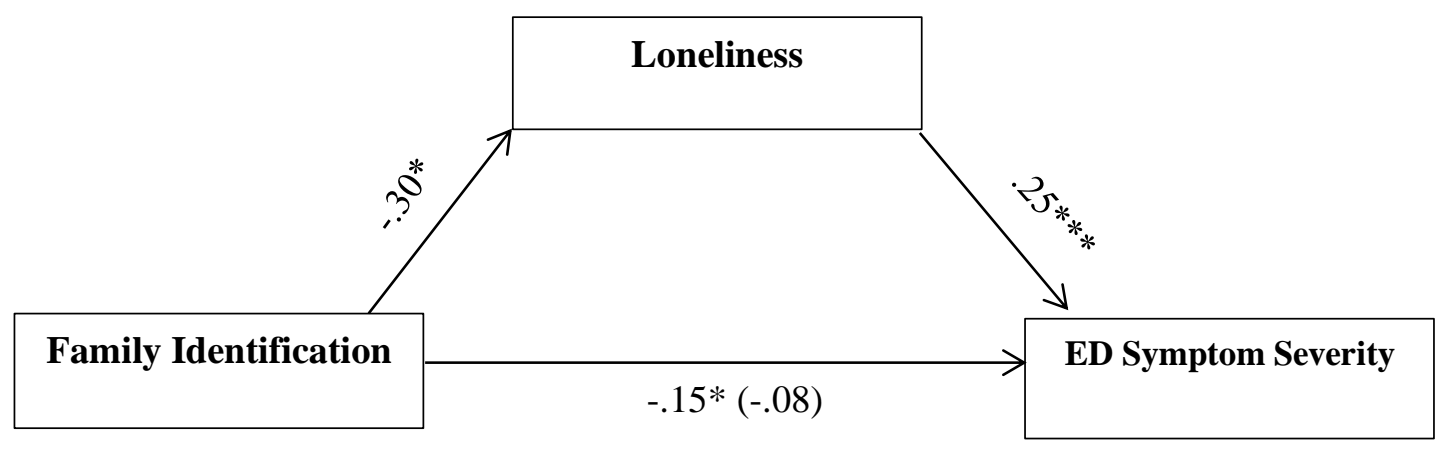

Figure 1. Study 1: Model depicting the indirect effect of family identification on symptom severity through loneliness. On the $c$ path, the value outside brackets is the total effect, while the value inside brackets is the direct effect. Control variables are not depicted. $* * * p<.001$, $* p<.05$. 


\section{Discussion}

As hypothesised, family identification was a significant negative predictor of ED symptom severity. We also observed our predicted mediation model, namely, strength of family identification was a negative predictor of loneliness, which itself was a positive predictor of ED symptom severity. This suggests that family identification may exert its benefits for PWED by protecting against loneliness. While we cannot draw firm conclusions on the directionality of the relationships, our results provide initial evidence for the association between the study variables. These are consistent with recent investigations of the association between group identification, loneliness, and health; however, this is the first study to provide evidence for this model among PWED or for ED symptomatology.

Nonetheless, our sample size for Study 1 was relatively small and restricted to those who were in contact with health services. Such contact might have incorporated family support and it is possible that such family support might indirectly assist in strengthening family identification. Therefore, our aim for Study 2 was to replicate this model with a larger and more diverse sample of PWED. We were also interested to explore whether this model would hold during a time when COVID-19 restrictions intensified the risk of loneliness for PWED. 


\section{Study 2}

Study 2 was conducted when social distancing measures were at their most stringent across the world (28 $8^{\text {th }}$ April- $3^{\text {rd }}$ June 2020). Mostly, people were only allowed to leave the house for exercise, essential shopping, and medicine. We posited that these measures would exacerbate ED symptoms, due to the specific ED-related concerns generated (e.g., food scarcity talk, the reduction in available support), as well as associated feelings of loneliness. It has been suggested that these restrictions may also lead to heightened anxiety among PWED (Fernández-Aranda et al., 2020) and so we were also interested in this as an outcome. Our aim for this study was to explore whether family identification was an important protective factor for PWED in the context of these stressors. We proposed a serial mediation model, such that family identification predicts reduced feelings of loneliness, which in turn predicts reduced ED-related impact of COVID-19, which in turn predicts reduced ED symptom severity and reduced anxiety.

\section{Participants and Procedure}

Two hundred and thirty-four participants (186 females, 8 males, 2 non-binary, 1 prefer not to say, 1 self-defined and 36 not reported; $M_{\text {age }}=28.92$ years, $S D=8.95$, age range $=18-77)$ took part. Participants were recruited online through advertisements on social media accounts and on the websites of mental health and ED charities in the UK, Ireland, and the USA. Most participants were from UK (34\%), USA (23\%) and Ireland (15\%). Of those who reported their living circumstances, $(71 \%)$ lived with family (parents/partner or spouse/other family members), $20 \%$ lived alone, and $9 \%$ lived with friends. At the start of data collection (28 ${ }^{\text {th }}$ April 2020, the UK had 3,473 confirmed cases, Ireland 386, and the USA 29,218 (World Health Organisation, 2020). 
Most participants (67\%) self-reported receiving an ED diagnosis. Of those, the most common self-reported diagnosis was Anorexia Nervosa (52\%), followed by OFSED (13\%), Combination AN/BN (11\%), Bulimia (8\%), Binge Eating Disorder (5\%), Avoidant/Restrictive Food Intake Disorder (2\%), Orthorexia (1\%), Overeating (1\%), and other disorders (7\%), which included individual participants reporting Anorexia Athletica, Anorexia Binge Purge Sub-Type, Anorexia with Exercise Addiction, Atypical Anorexia, nonpurging Bulimia Nervosa, and multiple diagnoses. Of those who indicated their treatment status, $46 \%$ were receiving treatment and $14 \%$ identified as fully recovered and no longer in treatment. Others reported having chosen to leave treatment $(20 \%)$ or only having contacted their GP with their concerns (20\%). Most had not contracted COVID-19 (77\%). 17 participants reported having suspected COVID-19, with one reporting a positive COVID-19 test.

\section{Measures}

Family identification and loneliness were measured in the same way as in Study 1.

Anxiety was measured with the seven-item anxiety sub-scale of the Depression, Anxiety, and Stress Scale (DASS-21; Lovibond \& Lovibond, 1995). Participants rated the frequency with which they had experienced each symptom in the previous seven days (e.g., "I was aware of dryness of my mouth") using a scale ranging from 0 ("Did not apply to me at all") to 3 ("Applied to me most of the time"). The sum of the items was calculated and multiplied by two, with higher values indicating stronger anxiety symptoms.

Eating disorder symptom severity was measured with Gideon et al.'s (2016) twelveitem Eating Disorder Examination Questionnaire-Short Form (EDE-QS). Participants were asked to rate the frequency with which they had experienced each symptom in the previous seven days (e.g., "Have you been deliberately trying to limit the amount of food you eat to 
influence your weight or shape (whether or not you have succeeded))?” on a scale ranging from 0 (“Zero days") to 3 (“6-7 days"). The sum of the items was found, with higher values indicating higher levels of eating disorder symptoms.

Eating disorder-related impact of COVID-19 was measured with a nine-item scale created for this study. Participants were asked to think about the previous two weeks in COVID-19 lockdown, and to rate their agreement with each item on a scale ranging from 1 (“Strongly Disagree”) to 7 ("Strongly Agree”): "I am worried about being able to access food items that form part of my meal plan"; "I am worried that an item of food is or will become limited"; "I am worried about limitations being placed on how often I can exercise"; "I am worried about limitations being placed on where I can exercise"; "Places I have sought support from (e.g., support groups, helplines) ae shut down during the pandemic"; "I have lost vital sources of support during the crisis"; "I have difficulty accessing my treatment team during the pandemic"; "I have difficulty accessing hospital or health services during the pandemic"; "Spending more time with others at home is stressful for me". The mean of the items was found, with higher values indicating higher impact.

As in Study 1, demographic information was also gathered, including age, gender, and whether the participant had received a formal eating disorder diagnosis. 


\section{Results}

\section{Descriptives and Correlations}

Descriptives and correlations for the variables can be found in Table 2. Family identification is below the mid-point, which is lower than typically observed in non-clinical samples. As anticipated, loneliness scores are particularly high and anxiety scores are in the severe clinical range. PWED who identified strongly with their family tended to feel less lonely $(p<.001)$, have fewer eating disorder symptoms $(p<.001)$, felt marginally less anxious $(p=.053)$, and felt marginally less impact of COVID-19 $(p=.051)$. These patterns remained when age, gender, and diagnosis (yes/no) were controlled for.

Table 2. Study 2: Means, standard deviations, alphas (where applicable), and correlations for the key variables

\begin{tabular}{|c|c|c|c|c|c|c|c|c|c|}
\hline Variable & Range & $M$ & $S D$ & $\alpha$ & 1 & 2 & 3 & 4 & 5 \\
\hline 1.Family Identification & $1-7$ & 3.56 & 1.89 & - & - & & & & \\
\hline 2.Loneliness & $3-9$ & 7.06 & 1.79 & .79 & $-.31 * * *$ & - & & & \\
\hline 3.ED Impact of COVID-19 & $1-7$ & 4.69 & 1.18 & .78 & $-.14 \dagger$ & $.39 * * *$ & - & & \\
\hline 4.Anxiety & $0-42$ & 15.79 & 10.01 & .86 & $-.13 \dagger$ & $.39 * * *$ & $.38 * * *$ & - & \\
\hline 5.ED Symptoms & 0.36 & 21.52 & 7.88 & .87 & $-.33 * * *$ & $.35 * * *$ & $.35 * * *$ & $.39 * * *$ & - \\
\hline
\end{tabular}

Note: $\dagger p<.054, * p<.05, * * * p<.001$ 


\section{Mediation Analyses}

Model 6 in version 3.4 of Hayes' (2017) PROCESS macro was used to test the serial mediation models.

Loneliness and ED-related Impact of COVID-19 Mediating the Relationship Between Family Identification and Anxiety

Supporting predictions (see Figure 2), there was a significant indirect effect of family identification on anxiety though loneliness and ED-related impact of COVID-19, Effect $=$ .14 , Boot $S E=.07$, Boot $L L C I=-.31$, Boot $U L C I=-.04$. Family identification was a negative predictor of loneliness, Coeff $=-.29, S E=.07, t=-4.41, p<.001, L L C I=-.42, U L C I=-.16$, while loneliness was a positive predictor of ED-related impact of COVID-19, Coeff $=.23, S E$ $=.04, t=5.25, p<.001, L L C I=.15, U L C I=.32$, and ED-related impact of COVID-19 was a positive predictor of anxiety, Coeff $=2.10, S E=.62, t=3.37, p=.0009, L L C I=.87, U L C I=$ 3.33. The total effect of family identification on anxiety was negative and significant, Effect $=$ $-.79, S E=.38, t=-2.09, p=.04, L L C I=-1.54, U L C I=-.05$, and this became non-significant when loneliness and ED-related impact of COVID-19 were accounted for (direct effect), Effect $=-.21, S E=.36, t=-.56, p=.57, L L C I=-.92, U L C I=.51$, indicating full mediation. 


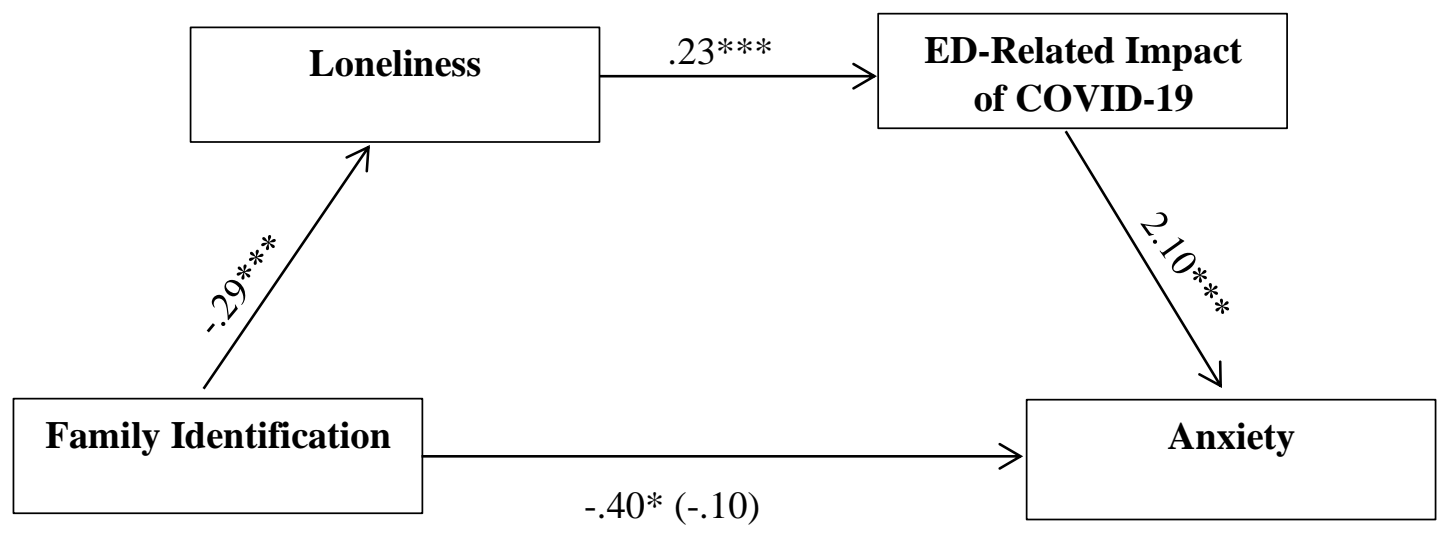

Figure 2. Study 2: Model depicting the indirect effect of family identification on anxiety through loneliness and ED-related impact of COVID-19. On the $c$ path, the value outside brackets is the total effect, while the value inside brackets is the direct effect. Control variables are not depicted. $* * * p<.001$.

Loneliness and ED-Related Impact of COVID-19 Mediating the Relationship Between Family Identification and ED Symptom Severity

Supporting predictions (see Figure 3), the analysis showed a significant indirect effect of family identification on ED symptom severity though loneliness and ED-related impact of COVID-19, Effect $=-.11$, Boot $S E=.05$, Boot $L L C I=-.23$, Boot $U L C I=-.03$. Family identification was a negative predictor of loneliness, Coeff $=-.29, S E=.07, t=-4.35, p<$ $.001, L L C I=-.42, U L C I=-.16$, while loneliness was a positive predictor of ED-related impact of COVID-19, Coeff $=.23, S E=.05, t=5.14, p<.001, L L C I=.14, U L C I=.32$, and ED-related impact of COVID-19 was a positive predictor of ED symptom severity, Coeff= $1.56, S E=.47, t=3.33, p=.001, L L C I=.64, U L C I=2.48$. The total effect of family identification on ED symptom severity was negative and significant, Effect $=-1.32, S E=.29$, $t=-4.57, p<.001, L L C I=-1.89, U L C I=-.75$, and this became weaker when loneliness and 
ED-related impact of COVID-19 were accounted for (direct effect), Effect $=-.91, S E=.28, t$ $=-3.26, p=.001, L L C I=-1.47, U L C I=-.36$, indicating partial mediation.

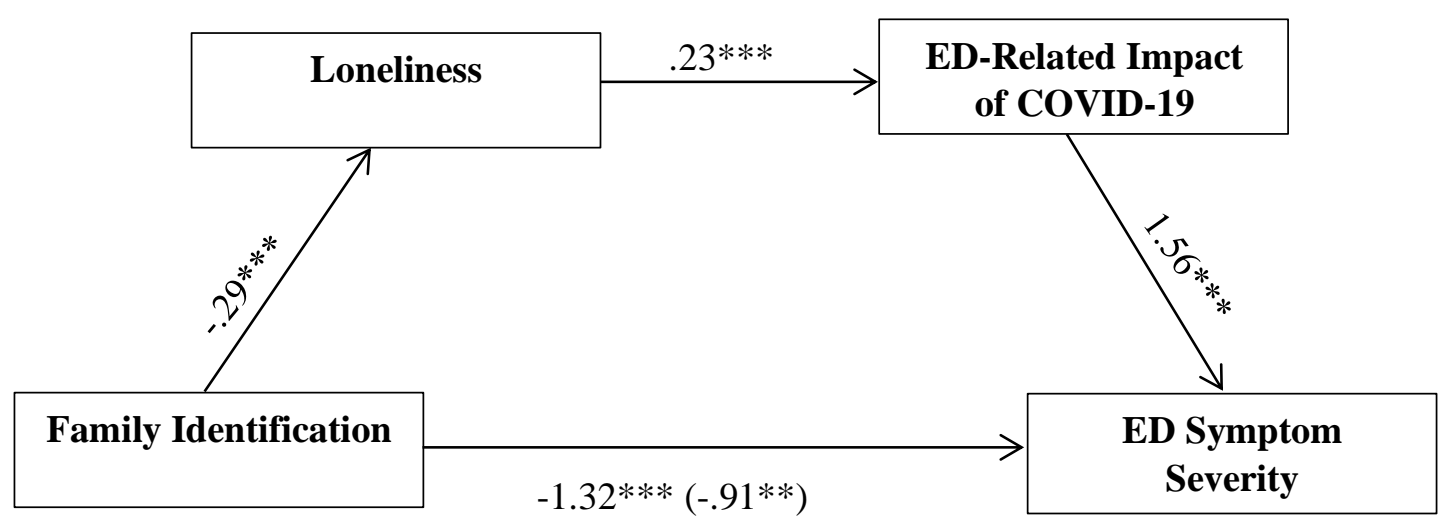

Figure 3. Study 2: Model depicting the indirect effect of family identification on ED symptom severity through loneliness and ED-related impact of COVID-19. On the $c$ path, the value outside brackets is the total effect, while the value inside brackets is the direct effect. Control variables are not depicted. $* * p<.01, * * * p<.001$.

\section{Discussion}

Study 2 replicated Study 1 findings, in the context of a larger and more diverse sample. Once again, family identification was a negative predictor of loneliness and ED symptom severity. There have been fears expressed by clinicians and PWED that the COVID-19 pandemic and, associated social distancing measures, may increase loneliness, and hence worsen ED symptoms (Fernández-Aranda et al., 2020). While this may be true, Study 2 found that family identification can play a key protective role, predicting reduced ED symptom severity and anxiety during the early stages of the COVID-19 pandemic. This was possible through its capacity to mitigate against loneliness and in turn, reducing fears of the ED-related impact of social distancing measures. This finding supports the important role of 
family in ED recovery. Furthermore, by incorporating the Social Identity Approach to Health (Haslam et al., 2018), this sheds light on how and why family are protective (i.e., through social identification and associated reductions in loneliness).

\section{General Discussion}

Recent years have seen increasing recognition of the valuable role that social connections play in ED recovery. Families are an important social recovery resource, however, prior research has lacked an established theoretical framework to explain why and how family group membership acts as a protective factor. In this article, we demonstrate the utility of the Social Identity Approach to Health (Haslam et al., 2018) as a framework for understanding the specific pathways through which the health benefits associated with family identification occur.

\section{Implications}

Our work is the first to provide evidence that family identification positively predicts mental health in PWED. Across two studies, we demonstrated that family identification predicted ED symptom severity. We also illustrated that family remains a protective factor in the context of an extreme event when the group is collectively under stress. We saw evidence that the COVID-19 pandemic is difficult for PWED. Levels of loneliness, anxiety, ED symptom severity and ED-related COVID impacts were all extremely elevated in Study 2. This supports other findings relating to the early impact of COVID-19 on PWED (FernándezAranda et al., 2020; Termorshuizen et al., 2020). Our work extends these findings to show a positive association between loneliness and the ED-related impact of COVID-19. Even in this challenging context, however, analyses revealed that family identification can be a protective factor. Our work provides a framework for understanding why increased connection with 
family during the pandemic can be a positive life change for PWED (Termorshuizen et al., 2020).

Furthermore, our findings provide a framework for understanding in general why family is so important in treating adults with an ED (Treasure \& Palazzo Nazar, 2016). They also support the focus in treatment on the family as a unit (a group) rather than as a collection of interpersonal relationships. The carers of adult PWED view a collaborative stance as more useful and report more positive caregiving experiences than with a directive stance (Geller et al., 2017). This fits with our assertion that the appropriate psychological process to focus on is family identification which looks at how a group collectively copes with an issue that affects all members. Family-based interventions that target the building of family identification to support a collaborative approach to tackling loneliness might be especially helpful. Social identification is malleable and responsive to context and intervention (e.g., Haslam et al., 2019; Steffens et al., 2019). Both samples were, on average, ambivalent in their level of family identification and these levels were lower than in typical samples (e.g., Stevenson et al., 2020), suggesting family identification could be increased with targeted intervention. This represents a novel approach to family intervention that does not require change to trait-type constructs. There can be a tendency to view isolation reductionistically as an individual factor needing biological recovery (Keys et al., 1950), but this study underlies the importance of tackling this issue through important social relationships.

\section{Strengths, Limitations, and Future Research}

This project had several strengths. One was the inclusion of two clinical samples, incorporating a total of over 300 PWED. Another strength was the capacity of these two studies in combination to demonstrate that the psychological processes under investigation are relevant both in general, and in the context of a major life changing stressor. Nonetheless, 
like all research, there are also limitations. First, both studies were cross-sectional and cannot establish causal relationships. Second, both study samples were predominantly female, White and cisgender. Consequently, males and minority ethnic communities are under-represented. Further investigation of these relationships is needed to confirm the generalisability of these findings. It would also be important to determine the factors that predict family identification to inform intervention development as well as investigating whether family identification operates in the same protective fashion for other family members.

\section{Conclusion}

In two studies, we demonstrated that family identification is protective for PWED. This was apparent in their reporting fewer and less severe ED symptoms, and in the context of the COVID-19 pandemic, reducing ED-related impact and reduced anxiety. In both studies, these benefits were attributable to the protective role of family identification against loneliness. This is consistent with a growing and persuasive body of research illustrating the toxic health consequences of loneliness outside of the context of EDs. These findings provide further support for the focus on the family in ED treatment, and specifically for conceptualising this in terms of one's subjective affiliation with, and self-definition in terms of, one's family (social identification). Furthermore, these findings highlight the role of the family as a critical protective resource both when it is "business as usual" as well as in times of crisis. 


\section{References}

Cardi, V., Mallorqui-Bague, N., Albano, G., Monteleone, A.M., Fernández-Aranda, F., \& Treasure, J. (2018). Social difficulties as risk and maintaining factors in Anorexia Nervosa. Frontiers in Psychiatry 9. doi: 10.3389/fpsyt.2018.00012

Clark Bryan, D., Macdonald, P., Ambwani, S., Cardi, V., Rowlands, K., Willmott, D., \& Treasure, J. (2020). Exploring the ways in which COVID-19 and lockdown has affected the lives of adult patients with anorexia nervosa and their carers. European Eating Disorders Review. https://doi.org/ 10.1002/erv.2762

Cockell, S.J., Zaitsoff, S.L., \& Geller, J. (2004). Maintaining change following eating disorder treatment. Professional Psychology: Research \& Practice, 35, 527-534. https://doi.org/ 10.1037/0735-0728.35.5.527

Cooper, M., Reilly, E.E., Siegel, J.A., Coniglio, K., Sadeh-Sharvit, S., Pisetsky, E.M., \& Anderson, L.M. (2020). Eating disorders during the COVID-19 pandemic and quarantining: An overview of risks and recommendations for treatment and early intervention. Eating Disorders: The Journal of Treatment \& Prevention. https://doi.org/10.1080/10640266.2020.1790271

Fernández-Aranda, F., Casas, M., Claes, L....Treasure, J. (2020). COVID-19 and implications for eating disorders. European Eating Disorders Review. https://doi.org/10.1002/erv.2738

Geller, J., Srikameswaran, S., Iyar, M., Zelichowska, J., Thibodeau, M., Brown, K. E., \& Dunn, E. C. (2017). Support stance in carers of adults with eating disorders: Factors associated with collaborative versus directive approaches. International Journal of Eating Disorders, 50(5), 498-505. 
Gideon, N., Hawkes, N., Mond, J., Saunders, R., Tchanturia, K., \& Serpell, L. (2016). Development and psychometric validation of the EDE-QS, a 12-item short form of the Eating Disorder Examination Questionnaire (EDE-Q). PLoS One, 13(11), $\mathrm{e} 0207256$.

Greenaway, K.H., Haslam, S.A., Cruwys, T., Branscombe, N.R., Ysseldyk, R., \& Heldreth, C. (215). From "we" to "me": Group identification enhances perceived personal control with consequences for health and wellbeing. Journal of Personality \& Social Psychology, 10, 53-74. https://doi.org/10.1037/pspi0000019

Haslam, C., Cruwys, T., Chang, M.X.-L., Bentley, S.V., Haslam, S.A., Dingle, G.A., \& Jetten, J. (2019). Groups 4 Health reduces loneliness and social anxiety in adults with psychological distress: Findings from a randomized controlled trial. Journal of Consulting \& Clinical Psychology, 87, 787-801. Advance online publication. https://doi.org/10.1037/ccp0000427

Haslam, C., Jetten, J., Cruwys, T., Dingle, G.A., \& Haslam, S.A. (2018). The new psychology of health: Unlocking the social cure. London: Routledge.

Hayes, A. F. (2017). Introduction to mediation, moderation, and conditional process analysis: A regression-based approach. London: Guilford Press.

Holt-Lunstad, J., Smith, T.B., Baker, M., Harris, T. \& Stephenson, D. (2015). Loneliness and social isolation as risk factors for mortality: A meta-analytic review. Perspectives on Psychological Science, 10, 227-237. https://doi.org/10.1177/1745691614568352

Hughes, M. E., Waite, L. J., Hawkley, L. C., \& Cacioppo, J. T. (2004). A short scale for measuring loneliness in large surveys: Results from two population-based studies. Research on Aging, 26(6), 655-672.

Keys, A., Brozek, J., Henshel, A., Mickelson, O., \& Taylor, H.L. (1950). The biology of human starvation (Vols. 1-2). Minneapolis, MN: University of Minneapolis Press. 
Le Grange, D., Lock, J., Loeb, K., \& Nicholls, D. (2010). Academy for eating disorders position paper: The role of the family in eating disorders. International Journal of Eating Disorders, 43, 1-5. https://doi.org/10.1002/eat.20751

Levine, M.P. (2012). Loneliness and eating disorders. The Journal of Psychology 146, 243 257.

Lovibond, S.H., \& Lovibond, P.F. (1995). Manual for the Depression Anxiety Stress Scales ( $2^{\text {nd }}$ ed.). Sydney: Psychology Foundation.

McNamara, N., Stevenson, C., Costa, S., Bowe, M., Wakefield, J., Kellezi, B., Wilson, I., Halder, M., \& Mair, E. (under review). Community identification, social support, and loneliness: The benefits of social identification for personal wellbeing. British Journal of Social Psychology.

McIntyre, J.C., Worsley, J., Corcoran, R., Harrison Woods, P., \& Bentall, R.P. (2018). Academic and non-academic predictors of student psychological distress: The role of social identity and loneliness. Journal of Mental Health, 27, 230-239. https://doi.org/10.1080/09638237.2018.1437608

McLaughlin, E. (2014). The EAT-16: Validation of a shortened form of the Eating Attitudes Test. Retrieved from https://digitalrepository.unm.edu/cgi/viewcontent.cgi?article=1093\&context=psy etds

McMaster, R., Beale, B., Hillege, S., \& Hagy, S. (2004). The parent experience of eating disorders: Interactions with health professionals. International Journal of Mental Health Nursing 13, 67-73.

Miller, K., Wakefield, J.RH., \& Sani, F. (2017). On the reciprocal effects between multiple group identifications and mental health: A longitudinal study of Scottish adolescents. 
British Journal of Clinical Psychology, 56, 357-371.

https://doi.org/10.1111/bjc.12143

Naughton, C.M., O’Donnell, A.T., Muldoon, O.T. (2015). Family identification: A beneficial process for young adults who grow up in homes affected by parental intimate partner violence. Frontiers in Psychology, 6:1249. https://doi.org/10.3389/fpsyg.015.01249

Patel, K., Tchanturia, K., \& Harrison, A. (2016). An exploration of social functioning in young people with eating disorders: A qualitative study. PLoS One 11, e0159910.

Perlman, D., \& Peplau, L.A. (1981). Towards a social psychology of loneliness. In S. Duck \& R. Gilmour (Eds.), Personal relationships in disorder. Academic Press.

Phillipou, A., Meyer, D., Neill, E., Tan, E.J., Toh, W.L., van Rheenan, T.E., \& Rossell, S.L. (2020). Eating and exercise behaviours in eating disorders and the general population during the COVID-19 pandemic in Australia: Initial results from the COLLATE project. International Journal of Eating Disorders. https://doi.org/10.1002/eat.23317

Postmes, T., Haslam, S. A., \& Jans, L. (2013). A single-item measure of social identification: Reliability, validity, and utility. British Journal of Social Psychology, 52(4), 597-617.

Reysen, S., Katzarska-Miller, I., Nesbit, S.M., \& Pierce, L. (2013). Further validation of a single-item measure of social identification. European Journal of Social Psychology, $43,463-470$.

Rodgers, R.F., Lombardo, C., Cerolini, S., Franko, D.L., Omori,M., Fuller-Tysczkiewicz, M., Linardon, J., Courtet, P. \& Guillaume, S. (2020). The impact of the COVID-19 pandemic on eating disorder risk and symptoms. International Journal of Eating Disorders. https://doi.org/10.1002/eat.23318 
Steffens, N.K., LaRue, C.J., Haslam, C., Walter, Z.C., Cruwys, T., Munt, K.A., Haslam, S.A., Jetten, J., \& Tarrant, M. (2019). Social identification-building interventions to improve health: A systematic review and meta-analysis. Health Psychology Review. Advance online publication. https://doi.org/10.1080/17437199.2019.1669481

Stevenson, C., Costa, S., Wakefield, J.R.H., Kellezi, B., \& Stack, R.J. (2020). Family identification facilitates coping with financial stress: A social identity approach to family financial resilience. Journal of Economic Psychology, 48. Article 102271. https://doi.org/10.1016/j/joep.2020.102271

Tajfel, H., \& Turner, J.C. (1979). An integrative theory of intergroup conflict. In W.G. Austin \& S. Worchel (Eds.), The social psychology of intergroup relations (pp.33-47). Brooks/Cole.

Termorshuizen, J.D., Watson, H.J., Thornton, L.M., Borg, S., Flatt, R.E., MacDermod, C.M., Harper, L.E., van Furth, E.F., Peat, C.M., \& Bulik, C.M. (2020). Early impact of COVID-19 on individuals with self-reported eating disorders: A survey of 1000 individuals in the United States and the Netherlands. International Journal of Eating Disorders. Advance online publication. https://doi.org/10.1002/eat.23353

Treasure, J., \& Palazzo Nazar, B. (2016). Interventions for the carers of patients with eating disorders. Current Psychiatry Reports, 18. Article no. 16. https://doi.org/10.1007/s11920-015-0652-3

Westwood, H., Lawrence, V., Fleming, C., Tchanturia, K. (2016). Exploration of friendship experiences, before and after illness onset in females with Anorexia Nervosa. PLoS One 11, e0163528.

World Health Organisation. (2020). WHO Coronavirus Disease (COVID-19) Dashboard. Retrieved from: https://covid19.who.int/ 
